\title{
Diseño de una actividad de aprendizaje interdisciplinar en red como propuesta para desarrollar las competencias de resolución de problemas, trabajo en equipo y comunicación efectiva
}

\author{
Ezequiel Godoy \\ ezgodoy@frro.utn.edu.ar \\ Facultad Regional Rosario \\ Universidad Tecnológica Nacional \\ Rosario, Argentina \\ Carina Fernández \\ carinafernandez@uncaus.edu.ar \\ Universidad Nacional del Chaco Austral \\ Pcia. Roque Sáenz Peña, Chaco, Argentina \\ Claudia M. Méndez \\ cmendez@fceqyn.unam.edu.ar \\ Facultad de Ciencias Exactas, Químicas y Naturales \\ Universidad Nacional de Misiones, \\ Posadas, Argentina \\ Mara C. Romero \\ mara@uncaus.edu.ar \\ Universidad Nacional del Chaco Austral - CONICET \\ Pcia. Roque Sáenz Peña, Chaco, Argentina \\ Noelia K. Villegas \\ nkvillegas@unsl.edu.ar \\ Facultad de Ingeniería y Ciencias Agropecuarias \\ Universidad Nacional de San Luis, \\ Villa Mercedes, San Luis, Argentina.
}

\section{RESUMEN}

La implementación de una educación universitaria basada en competencias requiere de estrategias de enseñanza y aprendizaje innovadoras que promuevan la formación articulada e interdisciplinaria de los estudiantes. El objetivo del trabajo es presentar una propuesta de aprendizaje colaborativo en red de estudiantes universitarios de carreras de ingeniería para el desarrollo de sus competencias de resolución de problemas, trabajo en equipo y comunicación efectiva. A los efectos, se describe la actividad de aprendizaje basado en problemas propuesta, incluyendo su contextualización, la estrategia de implementación, los recursos necesarios y los instrumentos de evaluación de la misma. Asimismo, se detallan dos casos de estudio en que se propone implementar dicha 
propuesta, abarcando carreras de ingeniería dictadas en cuatro universidades argentinas. Se espera que esta propuesta de aprendizaje colaborativo provea un encuadre para experiencias educativas interdisciplinares similares, orientadas al desarrollo de las competencias que los estudiantes universitarios necesitarán en su futura vida profesional.

Palabras clave: aprendizaje basado en problemas; trabajo en equipo; comunicación efectiva; articulación horizontal y vertical; formación por competencias. 


\title{
Design of an interdisciplinary network learning activity as proposal for developing the problem solving, teamwork and effective communication competencies
}

\begin{abstract}
The implementation of a competency-based university education highlights the need for innovative teaching and learning strategies that promote the articulated and interdisciplinary formation of students' knowledge, skills and attitudes. The objective of this work is to present a proposal for collaborative learning in a network of university students of engineering careers for the development of their problem-solving, teamwork and effective communication skills. In this sense, a problem-based learning activity proposal is described, including its contextualization, the implementation strategy, the required resources, and the evaluation instruments. Likewise, two case studies in which the proposal could be implemented are detailed, including engineering degree careers from four Argentine universities. It is expected that this collaborative learning proposal provides a framework for similar interdisciplinary educational experiences, aimed at developing the competencies that university students will need in their future professional lives.
\end{abstract}

Keywords: problem-based learning; teamwork; effective communication; horizontal and vertical articulation; competency-based education.

Artículo recibido: 15 noviembre. 2021 Aceptado para publicación: 10 diciembre 2021 Correspondencia: ezgodoy@frro.utn.edu.ar Conflictos de Interés: Ninguna que declarar 


\section{INTRODUCCIÓN}

El uso de metodologías de aprendizaje innovadoras implica que los estudiantes adquieran nuevas competencias interpersonales que les faciliten estrategias de interacción social y cooperación, como el aprendizaje colaborativo, que en la educación universitaria se presenta como una alternativa metodológica en la que los estudiantes tienen oportunidades recíprocas de aprender y enseñar a sus pares, lo que constituye un modelo de aprendizaje entre iguales (Guerra Santana, Rodríguez Pulido y Artiles Rodríguez, 2019). Esta metodología invita a los estudiantes a construir juntos, conjugando esfuerzos, talentos y competencias en busca del logro de las metas establecidas. Se caracteriza por basarse en una fuerte relación de interdependencia entre los miembros y una clara responsabilidad individual, exigiendo de los participantes habilidades comunicativas, relaciones simétricas y recíprocas y un deseo de compartir la resolución de las tareas, por lo que los equipos deben ser heterogéneos en cuanto a habilidades y características de sus miembros (Revelo-Sánchez, Collazos-Ordóñez y Jiménez-Toledo, 2018). La influencia del aprendizaje colaborativo en el logro de los desempeños y aprendizajes cognitivos, procedimentales y actitudinales en estudiantes de ciencias de la educación (Vargas et al., 2020), la aplicación de la metodología en entornos virtuales (Arena Madroñero, Tamez Almaguer y Lozano Rodríguez, 2017; Battaglia et al., 2019) y el efecto de aprendizaje colaborativo en el desarrollo de habilidades blandas (soft skills) (Lund y Aballay, 2020) son algunos ejemplos de las numerosas experiencias que han reportado los resultados de la implementación de esta forma de trabajo.

Las técnicas de aprendizaje cooperativo en el nivel educativo superior implican el desarrollo de estrategias de comunicación, competencias intelectuales y profesionales, y crecimiento personal, lo que da pie a la posibilidad de combinarlo con otras estrategias centradas en el aprendizaje. Por ello, el Aprendizaje Basado en Problemas (ABP) y la articulación interdisciplinaria resultan opciones de interés, por tratarse de metodologías que tienen puntos en común con el aprendizaje colaborativo en cuanto a las habilidades que buscan desarrollar. El ABP puede definirse como un método que promueve la habilidad para la resolución de problemas y la adquisición de conocimientos específicos, permitiendo la autorregulación del aprendizaje y propiciando, además, que los docentes actúen como guías o facilitadores que organizan y estimulan el proceso (Gil-Galván, 2018). A la vez, la articulación interdisciplinaria se refiere a la cooperación entre varias 
disciplinas en la búsqueda de la solución de un problema determinado, donde cada área aborda el proceso de acuerdo con los conocimientos propios de su especialización, generalmente en un campo de conocimiento común (Espinoza Freire, 2017), lo que aumenta la motivación de los estudiantes, disminuye el volumen de información a procesar y promueve un pensamiento más lógico, crítico, reflexivo e integrador (Villalonga-González, 2016). Es por ello que tanto el ABP (Fernández y Aguado, 2017; Morales Bueno, 2018; Rodríguez-Villalobos, Angulo-Sánchez y Leitón-Sancho, 2020) como la articulación interdisciplinar (Derka, 2020; Machado, Alvarez Dávila y Suarez, 2018; Pérez Castillo, Gradaille y Pérez Vilorio, 2018; Pozo Vinueza, Bodero Poveda y Cruz Pérez, 2017) han sido ampliamente utilizadas, especialmente en el marco del enfoque de formación por competencias en carreras de ingeniería.

La formación por competencias se centra en que el profesional graduado no sólo debe saber, sino también saber hacer y saber ser. En este sentido, el saber hacer no surge de la simple adquisición de conocimientos sino que es el resultado de una compleja estructura de conocimientos, habilidades y destrezas, que requiere ser reconocida expresamente en el proceso de aprendizaje para que la propuesta pedagógica incluya las actividades que permitan su desarrollo (CONFEDI, 2018), siendo relevante el rol de las metodologías activas para el logro de las competencias genéricas y transversales requeridas en los graduados universitarios en ingeniería (Kowalski et al., 2016).

Si bien desde hace tiempo la docencia universitaria experimenta cambios en cuanto a las estrategias de enseñanza y aprendizaje implementadas, la pandemia ha resaltado aún más la necesidad de repensar el itinerario formativo, ya que ha impactado tanto en docentes como estudiantes, quienes se han visto forzados a un viraje completo de la modalidad presencial a la virtual. Entre otras cuestiones, los docentes nos hemos visto obligados no sólo a revisar materiales y recursos didácticos que faciliten el aprendizaje. En cuanto a los estudiantes, las condiciones de aislamiento los han llevado a una "fatiga pandémica", caracterizada por tristeza, cansancio, pérdida de concentración, apatía, desmotivación, desorientación, inestabilidad e incertidumbre (Seglers, 2021), por lo que se debió atender a la necesidad de incorporar experiencias y modalidades de trabajo que permitan a los alumnos optar por niveles de profundización acordes con su propia motivación.

En este contexto, se describe aquí una propuesta de aprendizaje colaborativo en red de estudiantes universitarios que cursan carreras afines, mediante la implementación 
combinada del aprendizaje basado en problemas y la articulación interdisciplinaria como estrategias activas, con la finalidad de promover el desarrollo de las competencias de resolución de problemas, trabajo en equipo y comunicación efectiva, lo que podría motivar a los estudiantes para el logro de aprendizajes significativos, subsanando en parte los efectos de la denominada fatiga pandémica.

\section{ESTRATEGIAS METODOLÓGICAS}

\subsection{Encuadre: competencias, indicadores de desarrollo y resultados de aprendizaje}

Para diseñar una actividad activa de aprendizaje centrada en el estudiante, primero se deben definir las competencias que se desarrollarán, el nivel de alcance de dichas competencias (i.e. los indicadores de desarrollo), y los respectivos resultados de aprendizaje que orientan el proceso formativo (CONFEDI, 2014; Fernández March, 2010; Rossi, Forestello y Recabarren, 2020; Tardif, 2004). Puesto que las competencias son un saber ser o actuar complejo, su evaluación rigurosa y sistemática debe poner el acento sobre su grado de desarrollo y sobre los recursos que se deben movilizar. A los efectos, los indicadores de desarrollo asociados a las competencias permiten tomar en consideración esta complejidad en un contexto de formación y dar cuenta en un contexto de evaluación. Así, el concepto de competencias se completa con la formulación de los indicadores de desarrollo, que son concebidos como habilidades, procesos cognitivos, actitudes a aprender durante el desarrollo de una asignatura, y que contribuyen a la construcción de las competencias. Asimismo, permiten monitorear y evaluar el grado de adquisición y desarrollo de las competencias por parte del estudiante (Kennedy, 2007).

Se seleccionaron aquí tres competencias a desarrollar mediante la actividad propuesta, que se listan en la primera columna de la Tabla 1. En la segunda columna, se detallan los indicadores de desarrollo asociados a cada competencia, a los que se realizará seguimiento durante la actividad.

Se destaca que, con la progresiva implementación de una formación por competencias a lo largo de la currícula de las carreras de grado, se espera que los estudiantes hayan previamente comenzado a desarrollar en un nivel inicial algunas de las competencias aquí seleccionadas; aunque debido a la actual predominancia de los modelos de aprendizaje tradicionales, es posible que algunas cohortes carezcan de este tipo de formación.

Por su parte, los resultados de aprendizaje son enunciados acerca de lo que se espera que el estudiante sea capaz de saber, saber hacer y saber ser una vez terminado un proceso de 
aprendizaje [24]. Los mismos se componen de una acción a realizar, el objeto y finalidad de dicha acción, así como de las condiciones bajo las cuales se debe realizar, y deben ser redactados de forma que sean de fácil comprensión por parte de los alumnos.

Tabla 1. Competencias e Indicadores de Desarrollo

\begin{tabular}{|c|c|}
\hline Competencia & $\begin{array}{l}\text { Indicadores de Desarrollo } \\
\end{array}$ \\
\hline $\begin{array}{l}\text { Resolver } \\
\text { problemas }\end{array}$ & $\begin{array}{l}\text { - Delimitar el problema y formularlo de manera clara y precisa, } \\
\text { incluyendo el contexto particular en el análisis. } \\
\text { - Planificar una estrategia de búsqueda de soluciones: modalidad de } \\
\text { abordaje, tiempos requeridos, recursos necesarios, etc. } \\
\text { - Proponer alternativas de resolución al problema, incorporando las } \\
\text { dimensiones relevantes (tecnológica, económica, social y } \\
\text { medioambiental). } \\
\text { - Desarrollar criterios profesionales para la selección de la } \\
\text { alternativa de solución más adecuada en un contexto particular. }\end{array}$ \\
\hline $\begin{array}{l}\text { Trabajar en } \\
\text { equipo }\end{array}$ & $\begin{array}{l}\text { - Participar en la definición de la metodología de trabajo. } \\
\text { - Respetar los compromisos (tareas y plazos) contraídos con el } \\
\text { equipo. } \\
\text { - Identificar áreas de acuerdo y desacuerdo, y negociar para alcanzar } \\
\text { consensos. } \\
\text { - Promover un abordaje interdisciplinario al trabajo en equipo, } \\
\text { integrando las perspectivas de las diversas formaciones de los } \\
\text { integrantes. }\end{array}$ \\
\hline $\begin{array}{l}\text { Comunicarse } \\
\text { con } \\
\text { efectividad }\end{array}$ & $\begin{array}{l}\text { - Utilizar y articular de manera eficaz distintos lenguajes } \\
\text { - Usar eficazmente las herramientas tecnológicas apropiadas para la } \\
\text { comunicación. } \\
\text { - Exponer claramente ideas y conceptos a interlocutores diversos. }\end{array}$ \\
\hline
\end{tabular}
Fuente: Elaboración propia.

Asociados a las competencias genéricas y los indicadores de desarrollo seleccionados, que se listaron antes en la Tabla 1 , se proponen aquí los resultados de aprendizaje a alcanzar mediante la realización de la actividad propuesta:

RA1. Definir estrategias de solución integrales que permitan abordar problemas de ingeniería complejos, incorporando al diseño las dimensiones relevantes de su contexto específico.

RA2. Participar en las tareas acordadas por el equipo a los fines de desarrollar alternativas de solución factibles a problemáticas ingenieriles, considerando las implicaciones de diversos puntos de vista aportados desde diferentes áreas disciplinares.

RA3. Utilizar recursos y herramientas específicas que posibiliten la comunicación efectiva de las soluciones propuestas a destinatarios diversos, articulando las potencialidades que ofrecen los medios seleccionados. 
El logro de los resultados de aprendizaje propuestos se promoverá mediante la implementación del ABP, para lo cual, en primera instancia se debe diseñar una situación problemática que requiera de la toma de decisiones estratégicas por parte de los alumnos durante el proceso de búsqueda de una solución factible. Como recurso previo a la actividad, se requiere generar un marco amplio que permita incluir distintas instancias problemáticas desde la perspectiva de cada asignatura involucrada en la actividad. Cada situación problemática debe abarcar al menos una de las temáticas seleccionadas de cada asignatura involucrada, y debe asimismo ser contextualizada en el entorno social, económico y ambiental de la región o el país (Bur, 2012), de forma de movilizar a los estudiantes a poner en juego sus conocimientos, capacidades y habilidades para desarrollar una propuesta de solución.

\subsection{Diseño metodológico y contexto}

Como diseño metodológico, se empleará un estudio de casos (Yin, 2017), en el que se formarán redes de alumnos de diferentes universidades, evaluándose convergencias y divergencias que permitan una mejor interpretación de los resultados de la experiencia. Se realizará un estudio de corte transversal, en el cual, según la temporalización de las asignaturas implicadas, se prevén dos experiencias, una en el primer semestre del año y otra en el segundo, siendo las asignaturas participantes en cada instancia las que se muestran en la Tabla 2.

Tabla 2. Asignaturas en que se Implementará la Estrategia Propuesta

\begin{tabular}{|c|c|c|}
\hline Institución & Carrera & $\begin{array}{c}\text { Asignatura } \\
\text { (Año/Cuatrimestre) }\end{array}$ \\
\hline $\begin{array}{l}\text { Universidad Nacional del Chaco } \\
\text { Austral }\end{array}$ & Ingeniería en Alimentos & $\begin{array}{l}\text { Química Biológica } \\
\left(4^{\circ} / 1 \mathrm{c}\right)\end{array}$ \\
\hline $\begin{array}{l}\text { Facultad de Ciencias Exactas, } \\
\text { Químicas } \\
\text { Universidad Nacional de Misiones }\end{array}$ & $\begin{array}{l}\text { Ingeniería Química/ } \\
\text { Ingeniería en Alimentos }\end{array}$ & $\begin{array}{l}\text { Fisicoquímica II } \\
\left(3^{\circ} / 1 \mathrm{c}\right)\end{array}$ \\
\hline $\begin{array}{lr}\text { Facultad Regional } & \text { Rosario, } \\
\text { Universidad } & \text { Tecnológica } \\
\text { Nacional } & \\
\end{array}$ & Ingeniería Química & $\begin{array}{l}\text { Termodinámica } \\
(3 \% 1 \mathrm{c})\end{array}$ \\
\hline $\begin{array}{l}\text { Facultad de Ingeniería y Ciencias } \\
\text { Agropecuarias, Universidad } \\
\text { Nacional de San Luis }\end{array}$ & Ingeniería Química & $\begin{array}{l}\text { Balances de Materia } \\
\text { y Energía }\left(3^{\circ} / 1 \mathrm{c}\right)\end{array}$ \\
\hline $\begin{array}{l}\text { Universidad Nacional del Chaco } \\
\text { Austral }\end{array}$ & $\begin{array}{l}\text { Ingeniería Química/ } \\
\text { Ingeniería en Alimentos }\end{array}$ & $\begin{array}{l}\text { Fisicoquímica } \\
\left(3^{\circ} / 2 \mathrm{c}\right)\end{array}$ \\
\hline $\begin{array}{l}\text { Universidad Nacional del Chaco } \\
\text { Austral }\end{array}$ & Ingeniería en Alimentos & $\begin{array}{l}\text { Industrias } \\
\text { Alimentarias II } \\
\left(5^{\circ} / 2 \mathrm{c}\right)\end{array}$ \\
\hline
\end{tabular}




\begin{tabular}{lcl}
\hline $\begin{array}{l}\text { Facultad de Ingeniería y Ciencias } \\
\text { Agropecuarias, Universidad } \\
\text { Nacional de San Luis }\end{array}$ & $\begin{array}{c}\text { Ingeniería Química/ } \\
\text { Ingeniería en Alimentos }\end{array}$ & $\begin{array}{l}\text { Fenómenos de } \\
\text { Transporte }\left(3^{\circ} / 2 \mathrm{c}\right)\end{array}$ \\
\hline $\begin{array}{l}\text { Facultad de Ciencias Exactas, } \\
\text { Químicas y N Naturales, }\end{array}$ & $\begin{array}{c}\text { Ingeniería } \\
\text { Química/Ingeniería en } \\
\text { Universidad Nacional de Misiones }\end{array}$ & $\begin{array}{l}\text { Fisicoquímica I } \\
\left(2^{\circ} / 2 \mathrm{c}\right)\end{array}$ \\
\hline
\end{tabular}

Fuente: Elaboración propia.

Todas las asignaturas involucradas corresponden a las tecnologías básicas y aplicadas de las carreras de Ingeniería Química e Ingeniería en Alimentos. A esta altura de sus carreras, los estudiantes poseen conocimientos específicos previos en un nivel básico de balances de materia y transferencia de calor, así como conocimientos de ciencias básicas sobre matemáticas y química. En todos los casos se trabajará con todos los alumnos de las asignaturas involucradas.

\subsection{Enunciado de los problemas}

Se describen a continuación las situaciones problemáticas que se presentarán a los estudiantes para la implementación del ABP como parte de la metodología propuesta. Cada equipo de trabajo simulará ser una consultora en ingeniería de procesos, especializada en el diseño y optimización de procesos productivos de baja y mediana escala en la industria de alimentos, con el objeto de proveer asesoramiento especializado a productores nacionales.

\section{Problema 1, a utilizar en la instancia de la primera mitad de año}

La dueña de una pyme láctea, con una capacidad de procesamiento de 63000 litros de leche por día y ubicada en el centro de la provincia de Santa Fe, se pone en contacto con la consultora para solicitar asesoramiento en el diseño de una operación de producción de un nuevo yogurt adicionado con la cepa QB, que presenta similares características a la Bifidobacterium. Para ello, les solicita:

- Recomendar condiciones adecuadas para la fermentación del yogurt con la nueva cepa, que asimismo minimice el riesgo de contaminación del producto,

- Estimar la vida útil del producto, considerando las condiciones de comercialización que impone el mercado local,

- Definir la modalidad recomendada (batch o continua) para la producción del yogurt y cuantificar los requerimientos de materia y energía de dicha operación.

Problema 2, a utilizar en la instancia de la segunda mitad de año 
Un empresario aceitero desea aumentar la productividad de su línea de aceite de soja, por lo que requiere asesoría especializada a los fines de configurar una alternativa de proceso factible a los efectos. Asimismo, para testear nuevas condiciones operativas, el empresario informa que ha suscrito un acuerdo para utilizar una planta piloto de la zona, que tiene una línea de producción de 21,5 kg/h de aceite de soja. En este contexto, plantea las siguientes inquietudes sobre la operación de extracción por solvente:

- Considerando que usualmente la extracción se realiza en un rango de temperatura entre 50 y $60{ }^{\circ} \mathrm{C}$, ¿ es posible incrementar la temperatura operativa del extractor? ¿cómo impactará este cambio de temperatura sobre la vida útil del producto obtenido?,

- La adecuada operación del extractor de flujo continuo depende de las propiedades del aceite. ¿Se modifican las propiedades reológicas relevantes del aceite durante la extracción como consecuencia de la modificación de la temperatura de extracción?,

- Se utiliza hexano como solvente para la extracción, que se debe calentar desde la temperatura ambiente de almacenamiento hasta la temperatura de extracción. El calentamiento se realiza por intercambio con vapor generado en la caldera, que posee un rango operativo de 1 a 7 atm de presión. ¿Qué condiciones operativas se recomienda utilizar en la caldera, de forma de minimizar los costos operativos del proceso?

\subsection{Estrategia de implementación}

La estrategia de implementación de la actividad propuesta se presenta en la Fig. 1, donde se identifican tres etapas, que incluyen diferentes acciones orientadas a promover el desarrollo de las competencias seleccionadas.

\section{Primera etapa: Sesión virtual sincrónica inicial}

Presentación del problema: en una sesión virtual sincrónica, los docentes presentarán el problema al conjunto de todos los estudiantes que participan de la actividad. A continuación, se les solicitará identificar los aspectos del problema a cuya solución podrían aportar considerando los contenidos y competencias desarrollados en la asignatura que están cursando. También se les pedirá realizar una exploración inicial de la información que presumiblemente necesitarán para generar una propuesta de solución, así como de los resultados que se esperan obtener al final de la actividad. 
Organización del flujo de trabajo y conformación de los equipos: durante la misma sesión virtual sincrónica, se acordará entre alumnos y docentes el cronograma general de trabajo, programando las tareas a realizar por los alumnos de cada asignatura y los plazos de entrega de sus propuestas.

Figura 1. Estrategia de implementación de la propuesta.

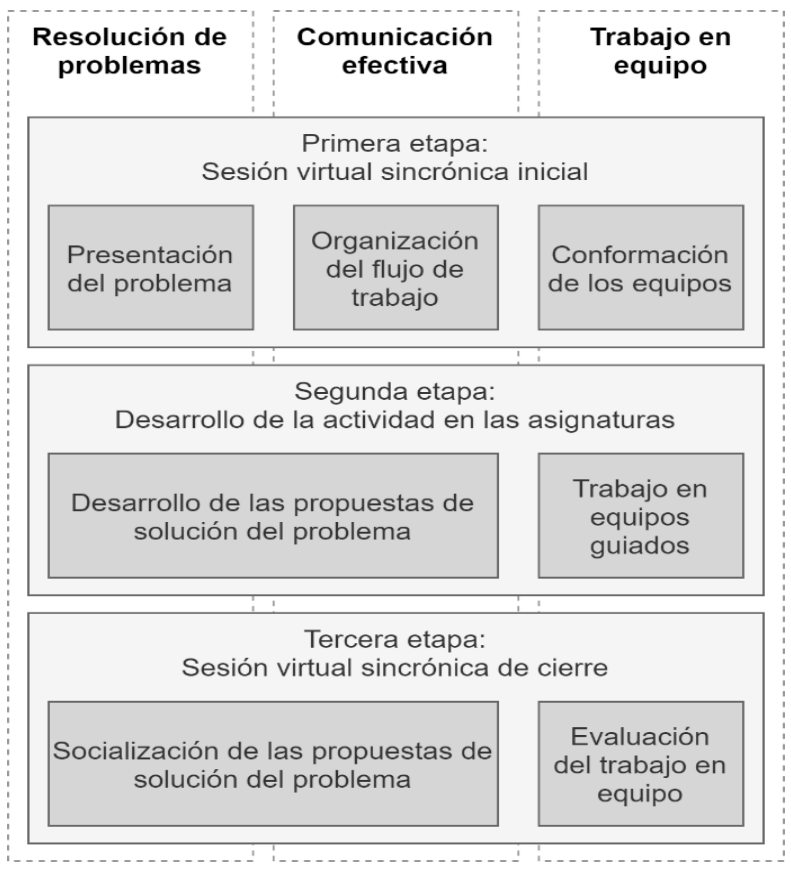

Fuente: Elaboración propia

\section{Segunda etapa: Desarrollo de la actividad en las asignaturas}

Desarrollo de las propuestas de solución del problema: los alumnos conformarán equipos interdisciplinarios asegurándose de contar con al menos 1 integrante de cada asignatura implicada. Cada equipo deberá realizar un análisis detallado del aspecto de la situación problemática; buscar y analizar información relevante sobre el problema, incluyendo el material de referencia que provean los docentes; plantear hipótesis de trabajo que permitan circunscribir el campo de acción; y proponer y desarrollar una alternativa factible de la situación problemática. Los equipos desarrollarán sus propuestas de solución en el repositorio online creado para la actividad, para facilitar el seguimiento y orientación por parte de los docentes.

Trabajo en equipos guiados: se adoptarán aquí los lineamientos de organización de equipos guiados (Collado Sevilla y Fachelli, 2019), que abarcan la etapa de consolidación y cohesión de los mismos, de forma que se favorezca la comunicación entre los 
integrantes y la implementación eficaz de las tareas planificadas para alcanzar los objetivos esperados. En este contexto, será necesario explicar a los equipos que deberán observar las siguientes pautas internas de funcionamiento:

- fijar sus objetivos y las tareas a realizar para alcanzarlos, especificando los responsables de llevar adelante cada tarea y los tiempos disponibles a los efectos,

- velar por el cumplimiento efectivo de estas pautas, expresando y discutiendo grupalmente las dificultades que pudieren surgir,

- mantener una comunicación fluida con sus compañeros, utilizando las herramientas y estrategias definidas en la propuesta.

Por su parte, los docentes tendrán la función de orientar el trabajo de los equipos, realizando sugerencias para mejorar el funcionamiento de los mismos cuando se detecte la necesidad o cuando alguno de los integrantes así lo requiera, lo que implica el compromiso docente de guiar a los estudiantes durante el trayecto recorrido.

\section{Tercera etapa: Sesión virtual sincrónica de cierre}

Socialización de las propuestas de solución del problema: en una sesión virtual sincrónica, cada equipo presentará su propuesta de solución a la situación problemática, debiendo abarcar todos los aspectos relevantes de la misma en forma sintética. Se generará también un espacio de intercambio en el que se discutirán las ventajas comparativas y potenciales dificultades de cada propuesta.

Evaluación del trabajo en equipo: los alumnos realizarán la autoevaluación y la evaluación de pares del trabajo en equipo.

\subsection{Estrategia de implementación}

Dadas las características de la metodología propuesta, la conectividad a internet resulta indispensable para su ejecución, mediante la utilización de los siguientes recursos:

- repositorio online, que permita compartir información y material entre todos los participantes de forma simple y directa. Se puede utilizar una carpeta compartida (Google Drive, Microsoft Onedrive), una página web (WordPress, Wix), o un aula virtual con acceso libre (Moodle).

- herramienta de comunicación sincrónica, para habilitar la realización de encuentros entre docentes y alumnos de todas las cátedras involucradas. Debido a la no presencialidad impuesta por la pandemia, se han popularizado opciones como Zoom 
y Google Meet, mientras que en general las universidades poseen sistemas de videoconferencia que se pueden utilizar desde las instituciones.

- herramienta de comunicación asincrónica, a los efectos de permitir la interacción entre los docentes y alumnos de todas las cátedras involucradas, intercambiar y difundir información, discutir inquietudes, entre otros. Como mensajería, una de las más conocidas es Slack, aunque hay nuevas opciones como Quill; mientras que también se pueden utilizar otras estrategias, como ser un foro configurado a los efectos.

- herramientas de trabajo colaborativo online, seleccionadas para facilitar el trabajo grupal de los equipos. En tal sentido, se pueden aprovechar las facilidades que proveen los documentos, planillas de cálculo y presentaciones online (Google Docs, Microsoft 365). Opcionalmente, se puede utilizar un gestor de proyectos (por ejemplo, Trello, Asana, entre otros), aunque solo se recomienda esta opción si la programación de las tareas de la actividad es algo más extensa.

\section{RESULTADOS Y DISCUSIÓN}

\subsection{Instrumentos de recolección de datos y de evaluación}

Para evaluar un trabajo colaborativo se debe contemplar tanto el producto final como el proceso de elaboración que llevó a desarrollar el mismo, por lo que resulta necesario contar con instrumentos de evaluación variados y metodológicamente planificados a los fines de promover una mejora continua de los procesos de aprendizaje.

Figura 2. Evaluación de la actividad

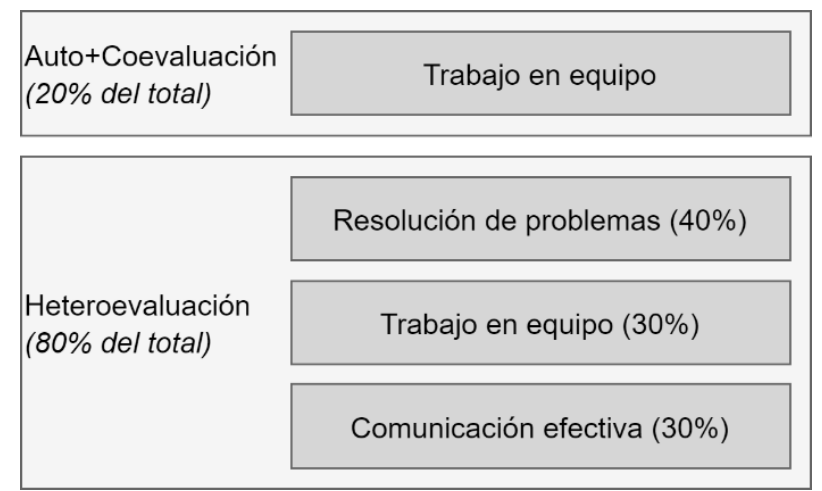

Fuente: Elaboración propia.

En la evaluación de la actividad, se propone entonces integrar dos instancias de evaluación: la autoevaluación y coevaluación por parte de los estudiantes del trabajo en equipo, y la heteroevaluación por parte de los docentes de la actividad en su conjunto. La 
calificación final en la actividad para cada estudiante estará constituida por la ponderación de sus calificaciones en ambas instancias, debiendo definirse los factores de ponderación en función de los pesos relativos que se desee dar a cada una, según la Fig. 2.

\subsection{Listas de cotejo}

La recolección cuantitativa de datos se realizará mediante la implementación de listas de cotejo, en las que se plasmarán las actividades realizadas por cada equipo y sus integrantes, tomando como base lo expuesto en (U. A. d. E. d. Hidalgo, 2019). La lista de cotejo recogerá datos de existencia de criterios y de frecuencia de los mismos, incluyéndose aspectos referidos al desempeño de cada equipo, como asistencia a tutorías y presentación de entregables, y también referidas al desempeño de cada estudiante, como intervenciones en los foros, aportes para el desarrollo de la actividad y actitud dentro del equipo.

\subsection{Autoevaluación y coevaluación del trabajo en equipo}

Mediante la autoevaluación, el estudiante evalúa sus propias actuaciones en un contexto determinado. Se busca así que los alumnos puedan reflexionar y tomar conciencia acerca de sus propios aprendizajes en el marco del trabajo en equipo y de los factores endógenos y exógenos que intervienen en ellos (París Mañas, Mas Torelló y Torrelles Nadal, 2016). Por su parte, la coevaluación es la valoración realizada entre pares mediante la cual se evalúan el desempeño y la calidad del trabajo en el equipo, así como el nivel de logro en relación a los objetivos de aprendizaje, entregando y recibiendo retroalimentación basada en criterios predefinidos (Jiménez-Tenorio et al., 2014).

\subsection{Heteroevaluación de la actividad}

Para la evaluación de la actividad a realizar por los docentes, se utilizará una rúbrica analítica, ya que este tipo de instrumento es útil para la valoración y autorregulación de la adquisición de competencias, al comunicar de forma efectiva a los estudiantes los aprendizajes esperados y sus correspondientes niveles de desempeño (Martínez, Arceo y Hurtado, 2016), así como también permitirles comprender más claramente qué son capaces de hacer y qué les falta aprender para llegar al siguiente nivel, y por lo tanto, porqué obtienen una determinada calificación (Martínez-Rojas, 2008). Se utiliza una rúbrica analítica a los efectos de desglosar el desempeño de los estudiantes en componentes o criterios, que se ponderan para luego configurar una calificación final. Al fijar los criterios, el mismo se puede subdividir de acuerdo con la profundidad requerida, 
definiendo el hecho y sin emitir criterios de subjetividad (Gatica-Lara y UribarrenBerrueta, 2013).

La rúbrica completa se presenta en el Anexo 1, e incluye todos los indicadores de desarrollo previamente listados en la Tabla 1 para las competencias seleccionadas. Para cada uno de dichos criterios, se definen cuatro niveles de desarrollo: Inadecuado, En desarrollo, Alcanzado, y Superado, que representan grados crecientes de adquisición de las dimensiones relevantes de cada competencia por parte de los alumnos.

La calificación definida por la rúbrica analítica se computa como la sumatoria del puntaje asociado al nivel de desarrollo alcanzado por el estudiante para un criterio/ indicador de desarrollo dado por el factor de ponderación de dicho criterio/ indicador de desarrollo.

\subsection{Evaluación del proceso}

Por otra parte, con la finalidad de obtener una retroalimentación del proceso, se realizará una encuesta de opinión, en la que los estudiantes podrán indicar las fortalezas y debilidades de la metodología propuesta como así también su preferencia o no frente a la enseñanza tradicional. El cuestionario a utilizar se dividirá en tres secciones: 1) presentación, donde se explicará la finalidad de la encuesta y se asegurará el anonimato al momento de responder, 2) sección destinada a caracterizar a la unidad de observación (incluyendo preguntas como edad, sexo, disponibilidad de recursos tecnológicos y conexión a internet, entre otros) y 3) lista de afirmaciones sobre el grado de acuerdo con aspectos referidos a la metodología empleada, el que se medirá con una escala Likert.

\subsection{Análisis de resultados}

Los datos se analizarán con herramientas de estadística descriptiva, mediante el cálculo de medidas de tendencia central y de dispersión.

\section{CONSIDERACIONES FINALES}

Con esta propuesta de aprendizaje colaborativo en red entre cinco asignaturas de diferentes carreras pertenecientes a distintas universidades, se espera que los estudiantes:

- Fortalezcan sus habilidades para la resolución de problemas de ingeniería, integrando de forma creativa los conocimientos de diferentes áreas temáticas.

- Mejoren su efectividad para expresarse de forma oral y escrita mediante la redacción de informes, discusión de alternativas y exposición de conclusiones.

- Desarrollen su capacidad y aptitud para trabajar de forma efectiva y colaborativa en equipos interdisciplinarios. 
En este sentido, se configura esta propuesta para ser implementada en asignaturas que se dicten simultáneamente en el mismo nivel y/o en diferentes niveles del trayecto formativo de carreras afines, debiendo considerarse la factibilidad de la articulación horizontal y/o vertical de contenidos y competencias entre las asignaturas involucradas y la posibilidad de formular resultados de aprendizaje que contribuyan a su desarrollo. Estas características permiten pensar en el diseño de futuras estrategias pedagógicas con un alcance más allá de lo institucional, por considerar que la metodología propuesta puede aplicarse en otras asignaturas y en otras universidades, siempre que se cuente con cantidades similares de alumnos.

La enseñanza centrada en el estudiante es un enfoque cada vez más utilizado en la educación superior, aplicándose en esta práctica diferentes métodos tendientes a promover aprendizajes más significativos, cambiando el rol del docente, quien se convierte en un facilitador del proceso de aprendizaje. Por ello, se espera que la implementación de esta propuesta contribuya al fortalecimiento de las competencias seleccionadas y se impulse la articulación de contenidos transversales y el trabajo en red entre diferentes universidades, promoviendo, además, en los estudiantes la reflexión sobre lo que se está aprendiendo y cómo se está aprendiendo, y en los docentes la toma de conciencia sobre los distintos estilos de aprendizaje a los fines de repensar las formas de enseñar y evaluar.

\section{LISTA DE REFERENCIAS}

Arena Madroñero, C., Tamez Almaguer, R. Lozano Rodríguez, A. (2017). Los estilos de aprendizaje y su relación con el aprendizaje colaborativo en cursos virtuales. Revista de Estilos de Aprendizaje, 10(20), 300-320. http://revistaestilosdeaprendizaje.com/article/view/1066

Battaglia, N., Neil, C., De Vincenzi, M., Beltramino, J. P. (2019). UAI case: desarrollo y evaluación de competencias en la ingeniería de software en un entorno virtual de aprendizaje colaborativo. XXI Workshop de Investigadores en Ciencias de la Computación (WICC 2019), San Juan, Argentina. http://sedici.unlp.edu.ar/handle/10915/77366

Bur, A. (2012). El Aprendizaje Basado en Problemas (ABP) en el aula universitaria. Reflexión Académica en Diseño y Comunicación, 119-121. https://fido.palermo.edu/servicios_dyc/publicacionesdc/archivos/379_libro.pdf 
Collado Sevilla, A. A., Fachelli, S. (2019). La competencia de trabajo en equipo: una experiencia de implementación y evaluación en un contexto universitario. REIRE Revista d'Innovació i Recerca en Educació, 12(2), 1-21. https://doi.org/10.1344/reire2019.12.222654

CONFEDI (2014). Competencias en Ingeniería. "Declaración de Valparaíso" sobre Competencias Genéricas de Egreso del Ingeniero Iberoamericano. Argentina: Universidad FASTA

Ediciones.

http://redi.ufasta.edu.ar:8080/xmlui/handle/123456789/409

CONFEDI (2018). Propuesta de estándares de segunda generación para la acreditación de carreras de ingeniería en la República Argentina "Libro Rojo de CONFEDI". Argentina: Universidad FASTA Ediciones. https://confedi.org.ar/publicacionesconfedi/

Espinoza Freire, E. (2017). Interdisciplinaridad un reto a la enseñanza superior. Revista Conrado, 13(60), 253-260. https://conrado.ucf.edu.cu/index.php/conrado/article/view/605

Derka, M., Fernández, C., Bedogni, G. Okulik, N. (2020). Articulación de espacios curriculares para el desarrollo de competencias en la enseñanza de Ingeniería Química. Revista Educación en Ingeniería, 15(29), 66-72. https://educacioneningenieria.org/index.php/edi/article/view/1074

Fernández C. L., Aguado, M. I. (2017). Aprendizaje basado en problemas como complemento de la enseñanza tradicional en Fisicoquímica. Educación Química, 28(3), 154-162. $\quad$ http://dx.doi.org/10.1016/j.eq.2017.03.001

Fernández March, A. (2010). La evaluación orientada al aprendizaje en un modelo de formación por competencias en la educación universitaria. Revista de Docencia Universitaria, 8(1), 11-34. https://doi.org/10.4995/redu.2010.6216

Gatica-Lara, F., Uribarren-Berrueta, T. D. N. J. (2013). ¿Cómo elaborar una rúbrica? Investigación en Educación Médica. 2(5), 61-65.

https://doi.org/10.1016/S2007-5057(13)72684-X

Gil-Galván, R. (2018). El uso del aprendizaje basado en problemas en la enseñanza universitaria: Análisis de las competencias adquiridas y su impacto. Revista Mexicana de Investigación Educativa, 23(76), 73-93. http://www.scielo.org.mx/pdf/rmie/v23n76/1405-6666-rmie-23-76-73.pdf 
Guerra Santana, M., Rodríguez Pulido J., Artiles Rodríguez, J. (2019). Aprendizaje colaborativo: experiencia innovadora en el alumnado universitario. Revista de Estudios y Experiencias en Educación, 18(36), 269-281. https://doi.org/10.21703/rexe.20191836guerra5

Kennedy, D. (2007). Redactar y utilizar resultados de aprendizaje: un manual práctico. Irlanda: University College Cork. https://intellectum.unisabana.edu.co/handle/10818/33010

Jiménez-Tenorio, N., Aragón, L., Sánchez, S., Aragón, C., Azcárate Goded, M. D. P., Cardeñoso, J. M., Moreno, F. (2014). La coevaluación-autoevaluación como instrumentos para valorar la competencia en el trabajo de equipo. IV Jornadas de Innovación Docente. Abriendo caminos para la mejora educativa, Sevilla. https://idus.us.es/handle/11441/59128

Kowalski, V., Posluszny, J. A., López, J. L., Erck, M., Enriquez, H. (2016). Formación por competencias en ingeniería: ¿Camino o destino? Revista Argentina de Ingeniería, 5(7), 130-141. https://confedi.org.ar/publicaciones/radi-no-7/

Lund, M. I., Aballay, L. N. (2020). Promoviendo el desarrollo de habilidades blandas en entornos distribuidos y colaborativos: una estrategia de enseñanza-aprendizaje en tiempos de pandemia. II Simposio Argentino de Educación en Informática (SAEI 2020), Buenos Aires, Argentina. http://sedici.unlp.edu.ar/handle/10915/116616

Machado, G., Alvarez Dávila, M., Suarez, S. (2018). Articulación nivel medio universidad: el laboratorio de química como nexo elector de una carrera de ingeniería. III Congreso Internacional de Enseñanza de las Ciencias Básicas CIECiBa 2018, Concordia. https://ria.utn.edu.ar/handle/20.500.12272/3401

Martínez, L. C. V., Arceo, F. D. B., Hurtado, J. C. T. (2016). Las rúbricas para la evaluación de competencias en la universidad bajo la lupa de profesores e investigadores. Congreso Internacional de Educación Evaluación 2016, Tlaxcala.

http://posgradoeducacionuatx.org/pdf2016/A128.pdf

Martínez-Rojas, J. G. (2008). Las rúbricas en la evaluación escolar: su construcción y su uso. Avances en Medición, 6(129), 38.

https://www.uaem.mx/sites/default/files/facultad-de medicina/descargas/construccion-yuso-de-rubricas-de-evaluacion.pdf 
Morales Bueno, P. (2018). Aprendizaje basado en problemas (ABP) y habilidades de pensamiento crítico, ¿una relación vinculante? Revista Electrónica Interuniversitaria de Formación Del Profesorado, 21(2), 91-108. https://doi.org/10.6018/reifop.21.2.323371

París Mañas, G., Mas Torelló, O., Torrelles Nadal, C. (2016). La evaluación de la competencia "trabajo en equipo" de los estudiantes universitarios. Revista d'Innovació Docent $\quad$ Universitària, $\quad 86$ 87. https://doi.org/10.1344/RIDU2016.8.10

Pérez Castillo, J., Gradaille, M. M., Pérez Vilorio, L. (2018). Las relaciones interdisciplinarias en los cursos por encuentro de la carrera Licenciatura en Educación Primaria. Revista Conrado, 14(61), 105-110. http://scielo.sld.cu/pdf/rc/v14n61/rc166118.pdf

Pozo Vinueza, M., Bodero Poveda, E., Cruz Pérez, M. (2017). La formación investigativa interdisciplinaria de los estudiantes universitarios con el empleo de las tecnologías de la información y la comunicación (TIC) y su dinámica. Academia \& Virtualidad, 10(1), 107-122. https://doi.org/10.18359/ravi.2670

Revelo-Sánchez, O., Collazos-Ordóñez C., Jiménez-Toledo, J. (2018). El trabajo colaborativo como estrategia didáctica para la enseñanzalaprendizaje de la programación: una revisión sistemática de la literatura. TecnoLógicas, 21(41), 115-134. $\quad$ http://www.scielo.org.co/pdf/teclo/v21n41/v21n41a08.pdf

Rodríguez-Villalobos, R., Angulo-Sánchez, L., Leitón-Sancho, N. (2020). Desarrollo de la metodología de aprendizaje basado en problemas en un curso de ingeniería. $\begin{array}{llll}\text { Educación en } & \text { Ingeniería, } & \text { 26-33. }\end{array}$ https://educacioneningenieria.org/index.php/edi/article/view/1122

Rossi, R. R., Forestello, R. P., Recabarren, P. G. (2020). Rediseño de un programa por competencias. El caso de Electrónica Digital I. Educación en Ingeniería, 15(29), 52-59. https://educacioneningenieria.org/index.php/edi/article/view/1071

Seglers, T. (2021). Fatiga pandémica en las universidades: «Hemos perdido los mejores años de la vida». El Diario de la Educación. https://eldiariodelaeducacion.com/2021/04/21/fatiga-pandemica-en-lasuniversidades-hemos-perdido-los-mejores-anos-de-la-vida/ 
Tardif, J. (2004). Un passage obligé dans la planification de l'évaluation des compétences: déterminer des indicateurs progressifs et terminaux de développement (2e partie). Pédagogie collégiale, 18(2), 13-20, 2004. http://eduq.info/xmlui/handle/11515/21545

U. A. d. E. d. Hidalgo (2019). Catálogo de Listas de Cotejo.

https://www.uaeh.edu.mx/division_academica/educacion-media/docs/2019/listas-decotejo.pdf

Vargas, K., Yana, M., Pérez, K., Chura, W., Alanoca, R. (2020). Aprendizaje colaborativo: una estrategia que humaniza la educación. Revista Innova Educación, 2(2), 363-379. $\quad$ https://doi.org/10.35622/j.rie.2020.02.009

Villalonga-González, M., Ugarte-Marchena, M., Aguirre-Azahares, N., García-Lora, R. (2016). Actividades interdisciplinarias en las asignaturas Ingeniería de Procesos I y Química General I en primer año de la carrera Ingeniería Química. Revista Cubana de Química, 28(1), 394-408. http://scielo.sld.cu/pdf/ind/v28n1/ind05116.pdf

Yin, R. (2017). Case study research and applications: design and methods. SAGE Publications. https://us.sagepub.com/en-us/nam/case-study-research-andapplications/book250150 
Anexo 1. Rúbrica de evaluación de la actividad.

\begin{tabular}{|c|c|c|c|c|c|c|}
\hline \multirow[b]{2}{*}{ Competencia } & \multirow{2}{*}{$\begin{array}{l}\text { Criterio (Factor } \\
\text { de ponderación) }\end{array}$} & \multirow{2}{*}{$\begin{array}{l}\text { Indicador de } \\
\text { Desarrollo }\end{array}$} & \multicolumn{4}{|c|}{ Nivel de Dominio (Puntaje) } \\
\hline & & & $\begin{array}{c}\text { Inadecuado } \\
\text { (1) }\end{array}$ & $\begin{array}{c}\text { En desarrollo } \\
\text { (4) }\end{array}$ & $\begin{array}{c}\text { Alcanzado } \\
\text { (7) }\end{array}$ & $\begin{array}{c}\text { Superado } \\
\text { (10) }\end{array}$ \\
\hline \multirow[t]{4}{*}{$\begin{array}{l}\text { Resolver } \\
\text { problemas }\end{array}$} & $\begin{array}{l}\text { Comprensión } \\
\text { del problema } \\
(10 \%)\end{array}$ & 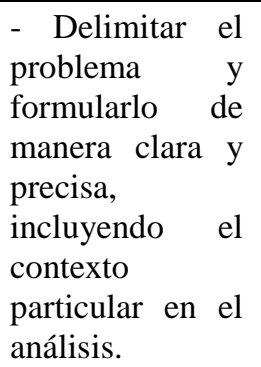 & \begin{tabular}{lr}
\multicolumn{2}{|l}{ No identifica las } \\
variables & de \\
diseño & y \\
operativas & \\
asociadas & al \\
problema. & \\
\end{tabular} & $\begin{array}{lr}\begin{array}{lr}\text { Identifica } \\
\text { algunas de }\end{array} & \text { solo } \\
\text { variables } & \text { de } \\
\text { diseño } & \text { y } \\
\text { operativas } & \\
\text { asociadas } & \text { al } \\
\text { problema. } & \\
& \end{array}$ & $\begin{array}{lr}\text { Identifica } & \\
\text { todas } & \text { las } \\
\text { variables } & \text { de } \\
\text { diseño } & \text { y } \\
\text { operativas } & \\
\text { relevantes } & \\
\text { asociadas } & \text { al } \\
\text { problema. } & \end{array}$ & $\begin{array}{l}\text { Define conceptual y } \\
\text { operacionalmente } \\
\text { todas las variables } \\
\text { de diseño y } \\
\text { operativas } \\
\text { relevantes asociadas } \\
\text { al problema. }\end{array}$ \\
\hline & $\begin{array}{l}\text { Estrategia } \\
\text { adoptada } \\
(10 \%)\end{array}$ & $\begin{array}{l}\text { - Planificar } \\
\text { estrategia } \\
\text { búsqueda de } \\
\text { soluciones: } \\
\text { modalidad de } \\
\text { abordaje, } \\
\text { tiempos } \\
\text { requeridos, } \\
\text { recursos } \\
\text { necesarios, etc. }\end{array}$ & $\begin{array}{lr}\text { No define } & \text { una } \\
\text { estrategia } & \text { de } \\
\text { búsqueda } & \text { de } \\
\text { soluciones } & \text { al } \\
\text { problema. } & \end{array}$ & $\begin{array}{lr}\text { Aplica } & \text { una } \\
\text { estrategia } & \text { de } \\
\text { búsqueda } & \text { de } \\
\text { soluciones, que no } \\
\text { le permite resolver } \\
\text { el problema. }\end{array}$ & $\begin{array}{l}\text { Aplica una } \\
\text { estrategia de } \\
\text { búsqueda de } \\
\text { soluciones, } \\
\text { considerando } \\
\text { el tiempo, } \\
\text { recursos } \\
\text { necesarios y } \\
\text { pasos a seguir } \\
\text { para resolver } \\
\text { el problema. }\end{array}$ & $\begin{array}{lr}\text { Aplica } & \text { una } \\
\text { estrategia } & \text { de } \\
\text { búsqueda } & \text { de } \\
\text { soluciones, } & \\
\text { considerando el } \\
\text { tiempo, recursos } \\
\text { necesarios y pasos a } \\
\text { seguir para resolver } \\
\text { el problema, } \\
\text { manteniendo un hilo } \\
\text { conductor. }\end{array}$ \\
\hline & $\begin{array}{l}\text { Alternativas de } \\
\text { resolución para } \\
\text { el problema } \\
(10 \%)\end{array}$ & $\begin{array}{l}\text { - Proponer } \\
\text { alternativas de } \\
\text { resolución al } \\
\text { problema, } \\
\text { incorporando las } \\
\text { dimensiones } \\
\text { relevantes } \\
\text { (tecnológica, } \\
\text { económica, y } \\
\text { social medioambiental } \\
\text { ). }\end{array}$ & $\begin{array}{l}\text { No presenta } \\
\text { ninguna } \\
\text { alternativa de } \\
\text { resolución } \\
\text { problema. }\end{array}$ & $\begin{array}{l}\text { Presenta } \\
\text { alternativa } \\
\text { resolución } \\
\text { problema } \\
\text { vinculada } \\
\text { únicamente } \\
\text { resultado } \\
\text { numérico. }\end{array}$ & $\begin{array}{l}\text { Presenta una } \\
\text { alternativa de } \\
\text { resolución al } \\
\text { problema, } \\
\text { teniendo en } \\
\text { cuenta } \\
\text { aspectos } \\
\text { técnicos, } \\
\text { económicos, } \\
\text { sociales y } \\
\text { medioambient } \\
\text { ales. }\end{array}$ & $\begin{array}{l}\text { Presentan más de } \\
\text { una alternativa de } \\
\text { solución al } \\
\text { problema, teniendo } \\
\text { en cuenta aspectos } \\
\text { técnicos, } \\
\text { económicos, } \\
\text { sociales y } \\
\text { medioambientales. }\end{array}$ \\
\hline & $\begin{array}{l}\text { Presentación de } \\
\text { la solución } \\
(10 \%)\end{array}$ & $\begin{array}{l}\text { - Desarrollar } \\
\text { criterios } \\
\text { profesionales } \\
\text { para la selección } \\
\text { de la alternativa } \\
\text { de solución más } \\
\text { adecuada en un } \\
\text { contexto } \\
\text { particular. }\end{array}$ & $\begin{array}{l}\text { No presenta la } \\
\text { resolución del } \\
\text { problema. }\end{array}$ & $\begin{array}{lr}\text { Presenta } & \text { la } \\
\text { solución } & \text { del } \\
\text { problema, } & \text { sin } \\
\text { explicar por qué es } \\
\text { la } & \text { mejor } \\
\text { alternativa. } & \end{array}$ & $\begin{array}{l}\text { Presenta la } \\
\text { solución del } \\
\text { problema, } \\
\text { explicando } \\
\text { por qué es la } \\
\text { mejor } \\
\text { alternativa. }\end{array}$ & $\begin{array}{l}\text { Presenta la solución } \\
\text { del problema, } \\
\text { justificando por qué } \\
\text { es la mejor } \\
\text { alternativa, así como } \\
\text { los motivos por los } \\
\text { que se descartaron } \\
\text { las demás } \\
\text { alternativas. }\end{array}$ \\
\hline $\begin{array}{l}\text { Trabajar } \\
\text { equipo }\end{array}$ & $\begin{array}{l}\text { Colaboración } \\
\text { en la definición } \\
\text { y en la } \\
\text { distribución } \\
\text { de las tareas } \\
\text { del trabajo en } \\
\text { equipo } \\
(7,5 \%)\end{array}$ & $\begin{array}{l}\text { - Participa en la } \\
\text { definición de la } \\
\text { metodología de } \\
\text { trabajo y en la } \\
\text { distribución de } \\
\text { tareas. }\end{array}$ & $\begin{array}{l}\text { No participa en } \\
\text { la definición de } \\
\text { la metodología } \\
\text { de trabajo. }\end{array}$ & $\begin{array}{l}\text { Aporta } \\
\text { parcialmente a la } \\
\text { definición de la } \\
\text { metodología de } \\
\text { trabajo. }\end{array}$ & $\begin{array}{l}\text { Participa en la } \\
\text { definición de } \\
\text { la } \\
\text { metodología } \\
\text { de trabajo. }\end{array}$ & $\begin{array}{lr}\text { Participa en } & \text { la } \\
\text { definición de } & \text { la } \\
\text { metodología } & \text { de } \\
\text { trabajo, teniendo en } \\
\text { cuenta } & \text { la } \\
\text { distribución } & \text { del } \\
\text { trabajo entre } & \text { los } \\
\text { integrantes } & \text { del } \\
\text { equipo. } & \end{array}$ \\
\hline
\end{tabular}




\section{Diseño de una actividad de ...}

Responsabilida d para el trabajo en equipo $(7,5 \%)$ compromisos (tareas y plazos) contraídos con el equipo.
- Respetar los
No respeta los compromisos contraídos.

(1)

Presenta fuera de Presenta tiempo actividades equipo. acordadas por

a Presenta a tiempo as tiempo las las actividades actividades acordadas por el el acordadas por equipo, mediante las el equipo, herramientas de mediante las trabajo colaborativo herramientas y promoviendo de trabajo actitudes similares colaborativo. en sus compañeros.

$\begin{array}{ll}\text { Actuación } & -\quad \text { Identificar } \\ \text { para afrontar } & \text { áreas de acuerdo } \\ \text { los conflictos } & \text { y desacuerdo, y } \\ \text { del equipo y } & \text { negociar para } \\ \text { su cohesión } & \text { alcanzar } \\ (7,5 \%) & \text { consensos. }\end{array}$

Provoca

conflictos.

Evita afrontar los Actúa

Percibe

los

conflictos y positivamente conflictos y actúa

observa con en la rápidamente para

pasividad. resolución de evitarlos y/o conflictos. solucionarlos.

Trabaja Intercambia ideas Intercambia Intercambia ideas y individualmente y experiencias ideas y experiencias

Fomento del - Promover un espíritu de abordaje equipo

\begin{tabular}{|c|c|c|}
\hline \multirow[t]{3}{*}{$\begin{array}{l}\text { Comunicarse } \\
\text { con } \\
\text { efectividad }\end{array}$} & $\begin{array}{l}\text { Utilización de } \\
\text { lenguaje } \\
\text { académico en la } \\
\text { redacción de } \\
\text { informes } \\
(10 \%)\end{array}$ & $\begin{array}{l}\text { - Utilizar y } \\
\text { articular de } \\
\text { manera eficaz } \\
\text { distintos } \\
\text { lenguajes. }\end{array}$ \\
\hline & Utilización & Usar \\
\hline & $\begin{array}{l}\text { los medios } \\
\text { necesarios para } \\
\text { comunicar } \\
\text { ideas de forma } \\
\text { efectiva } \\
(10 \%)\end{array}$ & $\begin{array}{l}\text { eficazmente las } \\
\text { herramientas } \\
\text { tecnológicas } \\
\text { apropiadas para } \\
\text { la } \\
\text { comunicación. }\end{array}$ \\
\hline
\end{tabular}

Presentación oral de claramente ideas información y conceptos a técnica $(10 \%)$ interdisciplinari o al trabajo en equipo, integrando las perspectivas de las diversas formaciones de los integrantes.

, sin interactuar individuales, sin experiencias individuales,

con los demás considerar los individuales, integrándo los miembros del puntos de vista de considerando puntos de vista de equipo. los demás los puntos de todos los miembros miembros del vista de los del equipo. equipo. demás miembros del equipo.

No utiliza Utiliza recursos Utiliza

recursos gráficos y medios recursos

gráficos

ni adicionales de gráficos apoyo a la medios Utiliza recursos gráficos y medios medios adicionales de comunicación, que adicionales de generando un buen apoyo para una no ayudan al apoyo a la soporte para el comunicación análisis efectiva. interpretación.

e comunicación, análisis, que ayudan al interpretación y análisis e comunicación. interpretación.

\section{No utiliza las Utiliza herramientas tecnológicas \\ las Utiliza eficazmente las} propuestas para propuestas para el herramientas el aporte de aporte de ideas tecnológicas ideas en el fuera del contexto propuestas contexto del del problema. problema. interlocutores diversos. No

\section{Exponer No} conceptos referidos problema.

$$
\text { problema. }
$$

logra Expresa las ideas y Utiliza lenguaje

al (Expresa) con terminología específica para transmitir ideas conceptos referidos problema. al problema.
Utiliza efectiva $y$ activamente las herramientas tecnológicas para el aporte y discusión de ideas relevantes y atractivas en el contexto del el de ideas en el contexto
contexto del problema.

un Utiliza un lenguaje Expresa de manera convincente y con terminología específica al ir expresar las ideas y y conceptos referidos al problema.

al Expone con un lenguaje 
Godoy y otros

Presentación

oral información

técnica

$(10 \%)$
Exponer

claramente ideas y conceptos interlocutores diversos. $\mathrm{y}$ conceptos conceptos referidos problema. al referidos problema lenguaje coloquial. convincente específico.

$\mathrm{y}$

as Expone las ideas y

y conceptos referidos al problema con un

ideas al conceptos

en referidos al lenguaje especifico problema con y adaptado a los terminología interlocutores. específica.

Fuente: Elaboración propia. 